\title{
Temperature hysteresis of the order-disorder transition in carbon-supersaturated $\alpha$-Fe
}

\author{
P. Maugis,,${ }^{1, *}$ F. Danoix, ${ }^{2}$ H. Zapolsky, ${ }^{2}$ S. Cazottes,${ }^{3}$ and M. Gouné ${ }^{4}$ \\ ${ }^{1}$ Aix-Marseille Université, Université Toulon, CNRS, IM2NP, Marseille, France \\ ${ }^{2}$ Normandie Université, UNIROUEN, INSA Rouen, CNRS, Groupe de Physique des Matériaux, Rouen, France \\ ${ }^{3}$ Université de Lyon, INSA Lyon, MATEIS-CNRS, Lyon, France \\ ${ }^{4}$ ICMCB-CNRS, Pessac, France \\ (Received 12 October 2017; revised manuscript received 28 November 2017; published 12 December 2017)
}

\begin{abstract}
Although many works have been devoted to the order-disorder transition in carbon-supersaturated $\alpha$-Fe, all seem to have overlooked the temperature hysteresis phenomenon occurring around the critical temperature. It is shown, from a mean-field model based on the elasticity theory of point defects, that the origin of the temperature hysteresis is thermodynamic. As a consequence, both the critical temperature and carbon concentration for the order-disorder transition can be defined upon heating and cooling. The results obtained were successfully compared to molecular dynamics simulations, and are evidence that the transition is of first order and that linear elasticity is the predominant source of the thermodynamics of the $\mathrm{Fe}-\mathrm{C}$ solid solutions.
\end{abstract}

DOI: 10.1103/PhysRevB.96.214104

\section{INTRODUCTION}

In 1926, Fink and Campbell were the first to show that quenched carbon steels contain a phase with a tetragonal crystal structure [1]. The lattice of this so-called $\alpha^{\prime}$-martensite phase is tetragonally distorted, in contrast to the equilibrium body-centered-cubic (bcc) structure $\alpha$-Fe. The structure formed acquires the body-centered-tetragonal (bct) structure, with a corresponding $c / a$ axial ratio, $c$ being the long axis of the elongated cube and $a$ being its short axis.

Tetragonality is a direct consequence of carbon ordering onto one of the three possible subsets of octahedral sites of the bcc structure. The occurrence of the ordered phase was explained by two different mechanisms at least. The displacive transformation character of the face-centered-cubic (fcc) austenite to $\alpha^{\prime}$-martensite, and the associated Bain's deformation, impose that the octahedral interstitial sites of the parent fcc lattice transform into the interstitial sites of only one of the three octahedral sublattices of the bcc (or bct) structure. In that case, the tetragonal distortion is the product of the reconstruction process itself. This ordering can also be viewed as a self-induced preferential distribution of solute atoms in one of the three types of tetragonal interstitial positions. This approach, based on thermodynamic considerations (the ordered phase being more stable than the disordered one) is commonly referred to as Zener ordering, and is mainly driven by elastic interactions between carbon atoms [2]. Based on this idea, Khachaturyan studied carbon atoms ordering using a mean-field approximation [3]. A few years later, he proposed a more fundamental approach based on static concentration waves and microscopic elastic theory [4]. These models, and others [5-8], enable us to define the critical temperature $T_{c}$ of carbon ordering as

$$
T_{c}=0.361 \frac{\lambda_{0}}{k_{B}} u_{\mathrm{C}},
$$

\footnotetext{
*philippe.maugis@im2np.fr
}

where $u_{\mathrm{C}}$ is the $u$-fraction of carbon (ratio $\mathrm{C} / \mathrm{Fe}$ ), $k_{B}$ is Boltzmann's constant, and $\lambda_{0}$ is the strain interaction parameter.

Numerous theoretical studies based on different approaches were devoted to determining the value of $\lambda_{0}$. However, no clear consensus emerges since the calculated values of $\lambda_{0}$ ranged from a lower limit of $2.73 \mathrm{eV} /$ atom [4] to an upper limit of $10.7 \mathrm{eV} /$ atom [3]. This leads to much uncertainty regarding the calculated critical carbon concentration above which the tetragonal phase is stable at room temperature (ranging between 0.33 and 2.56 at. \%). Regarding the experimental results, the determination of the critical temperature as a function of carbon content is constrained by many physical phenomena, such as carbon segregation on martensite defects at low temperature and carbide precipitation at temperatures higher than $100^{\circ} \mathrm{C}$. Furthermore, the lattice parameters at room temperature and thus the tetragonality induced by carbon ordering onto one of the three possible octahedral sublattices are very difficult to measure even by advanced techniques [9,10]. Finally, there are few available experimental values of the critical carbon concentration of the cubic to tetragonal transition at room temperature. The existing ones range from 0.93 at. $\%[11,12]$ to 2.8 at. $\%$ [13].

The literature survey thus shows that the mechanisms of both martensite formation and order-disorder transition require further exploration although two recent works based on molecular dynamic calculations and density functional theory provide two main clarifications [6,14]. First, the mechanism of ordering would depend on both cooling rate and carbon content. Indeed, for concentrations above $x_{\mathrm{Cmax}}=3.8$ at. $\%$, a Bain-type transformation from fcc austenite to the ordered martensite takes places [6]. For concentrations below $x_{\mathrm{Cmax}}$, a diffusion-based transformation is proposed [14]. The disordered phase has to be ordered by atomic diffusion during the quench of fcc austenite. This diffusive regime would be limited by the cooling rate that must be slow enough, and by critical temperature and concentration (respectively, $273 \mathrm{~K}$ and 1.03 at. \%) below which no martensite formation is expected due to the slow diffusivity of carbon atoms. Second, a hysteresis in ordering of carbon on heating and cooling was very recently highlighted and was attributed to 
kinetic effects [14]. Indeed, upon cooling from a disordered structure, it was suggested that a carbon atom makes several atomic jumps to order, while the process of disordering upon heating could be accomplished simply by one single atomic jump.

It is in this context that our work is undertaken. A mean-field model, based on the elasticity theory of point defects, is developed. From the Gibbs energy function of disordered $\alpha-\mathrm{Fe}$ and partially ordered $\alpha^{\prime}$-Fe, the temperature hysteresis of the order-disorder transition is highlighted. The results obtained, successfully compared with those obtained by Sinclair et al. [14] using molecular dynamic simulations, give some clarifications regarding the origin of the temperature hysteresis, the order of the transition, and the elastic contribution to the thermodynamics of $\mathrm{Fe}-\mathrm{C}$ alloys.

\section{MODEL}

Martensite and ferrite phases are both represented by three octahedral sublattices for carbon atoms in a bct-Fe host lattice. In this view, ferrite is the bcc disordered state of martensite, where the three sublattices are equally and randomly occupied by carbon atoms. Accordingly, the chemical formula of martensite in the compound formalism [15] is $\mathrm{Fe}(\mathrm{C}, \mathrm{Va})_{1}(\mathrm{C}, \mathrm{Va})_{1}(\mathrm{C}, \mathrm{Va})_{1}$. The three octahedral sublattices are labeled 1,2 , and 3 . The $u$-fractions of carbon on the octahedral sublattices are, respectively, $u_{1}, u_{2}$, and $u_{3}$. For instance, $u_{i}$ is the number of carbon atoms on sublattice $i$, per iron atom of the crystal. The total $u$-fraction of carbon is $u_{\mathrm{C}}=u_{1}+u_{2}+u_{3}$ and the molar fraction is $x_{\mathrm{C}}=u_{\mathrm{C}} /\left(1+u_{\mathrm{C}}\right)$.

The expression of the Gibbs energy $G$ of the alloy as a function of the $u$-fractions is needed in order to express the equilibrium state as the minimum of $G$. In view of the discussion presented in the next section of the paper, it is useful to recall here the premises and hypotheses underlying the mean-field approach. Following Zener [2], the enthalpy $H$ of the crystal is mostly of elastic origin, resulting from the interactions of the elastic fields created by each carbon atom. The enthalpy has two contributions: the self-energy of the strained lattice and the carbon-strain interaction energy. Density-functional theory (DFT) calculations show that the elasticity coefficients of Fe-C martensite are almost composition independent up to high carbon concentrations (11 at. \%) [16], and that the lattice parameters are linear functions of the carbon content [17]. These findings justify the use of the linear elasticity theory of point defects in the context of this paper.

Let us define $E_{\text {int }}$ as the interaction energy of a carbon atom with a local deformation field $\varepsilon^{d} . \varepsilon^{d}$ is the deformation relative to pure, undeformed bcc Fe. The deformation field may be created by point defects (vacancies, solute atoms),
TABLE I. Elastic dipole constants [Eq. (2)] and expansion concentration coefficients [Eq. (3)] from the literature.

\begin{tabular}{lcrrr}
\hline \hline & $P_{a}(\mathrm{eV})$ & $P_{c}(\mathrm{eV})$ & \multicolumn{1}{c}{$\delta_{a}$} & \multicolumn{1}{c}{$\delta_{c}$} \\
\hline Cheng et al. [20] & & & -0.094 & 0.85 \\
Clouet et al. [19] & 3.40 & 8.03 & -0.088 & 0.56 \\
Chentouf et al. [17] & 8.03 & 15.04 & -0.025 & 0.84 \\
\hline \hline
\end{tabular}

by structural defects (dislocations, grain boundaries), or by internal and applied stresses. $E_{\text {int }}$ is the opposite of the carbondefect binding energy $E_{b}$. According to [18,19], $E_{\text {int }}=-P$ : $\varepsilon^{d}$, where $P$ is the elastic dipole tensor. For a carbon atom in an octahedral site of type 3 , the elastic dipole recalls the tetragonal symmetry of the corresponding interstitial sites; it is written as

$$
P^{(3)}=\left(\begin{array}{ccc}
P_{a} & 0 & 0 \\
0 & P_{a} & 0 \\
0 & 0 & P_{c}
\end{array}\right)
$$

Equivalent definitions stand for $P^{(1)}$ and $P^{(2)}$ by index permutation. In the absence of external stresses, the elastic deformation $\varepsilon^{d}$ is produced solely by the set of carbon atoms in the crystal. In the mean-field approximation, it is the sum of the deformations induced by each individual carbon atom, independently of their positions. For instance, the carbon atoms sitting on the type- 3 sites contribute to the total deformation by $\varepsilon^{(3)}=\delta^{(3)} u_{3}$ with

$$
\delta^{(3)}=\left(\begin{array}{ccc}
\delta_{a} & 0 & 0 \\
0 & \delta_{a} & 0 \\
0 & 0 & \delta_{c}
\end{array}\right) .
$$

The trace of tensor $\delta$ is site independent, and is related to the carbon-induced volume expansion. The lattice expansion concentration coefficients $\delta_{a}$ and $\delta_{c}$ are related to Vegard's law and can be measured from experimental data [20,21], computed by molecular dynamics [19], or computed $a b$ initio [17] (see Table I). The agreement of DFT values [17] with experiment is very good for $\delta_{c}$. Although the relative error for $\delta_{a}$ is large, the absolute error (0.07) is in the range of the uncertainty of the method. These values will be used in this section for study of the ordering behavior. The data of Clouet et al. [19] were computed from the same potential as in the molecular dynamics (MD) simulations of Sinclair et al. [14]. They will be used in the discussion for comparison of our model against molecular dynamics.

The total deformation induced by the set of carbon atoms is the sum of contributions of each sublattice:

$$
\varepsilon^{d}=\left(\begin{array}{ccc}
\left(u_{2}+u_{3}\right) \delta_{a}+u_{1} \delta_{c} & 0 & 0 \\
0 & \left(u_{1}+u_{3}\right) \delta_{a}+u_{2} \delta_{c} & 0 \\
0 & 0 & \left(u_{1}+u_{2}\right) \delta_{a}+u_{3} \delta_{c}
\end{array}\right)
$$

The total interaction energy per iron atom is the sum of contributions of each sublattice $i: H_{\text {int }}=\sum_{i} u_{i} P^{(i)}: \varepsilon^{d}$. At mechanical equilibrium, the unconstrained lattice will adopt a state of strain such as to minimize the total elastic energy 
TABLE II. Strain interaction parameters defined in Eqs. (6) and (9). Energies are in $\mathrm{eV}$.

\begin{tabular}{lllll}
\hline \hline & $h_{a}$ & $h_{c}$ & $h_{X}$ & $h_{\eta}$ \\
\hline Clouet et al. [19] & 0.449 & 1.95 & 0.949 & 1.00 \\
Chentouf et al. [17] & 3.09 & 6.21 & 4.13 & 2.08 \\
\hline \hline
\end{tabular}

of the system. According to Bacon [18], this occurs when the enthalpy verifies $H=\frac{1}{2} H_{\text {int }}$. Hence the enthalpy per iron atom is written, as a function of the $u_{i}$ 's,

$$
H\left(\left\{u_{i}\right\}\right)=-h_{c} \sum_{i}\left(u_{i}\right)^{2}-2 h_{a} \sum_{i<j} u_{i} u_{j},
$$

where two strain interaction parameters have been introduced (see Table II):

$$
\begin{aligned}
h_{a} & =\frac{1}{2}\left(P_{a} \delta_{a}+P_{a} \delta_{c}+P_{\mathrm{c}} \delta_{a}\right) \\
h_{\mathrm{c}} & =\frac{1}{2}\left(2 P_{a} \delta_{a}+P_{\mathrm{c}} \delta_{c}\right) .
\end{aligned}
$$

The quadratic form of the enthalpy as function of composition $u_{\mathrm{C}}$ in Eq. (5) arises from the interaction of the strain field, linear in $u_{\mathrm{C}}$, with the population of carbon atoms of concentration $u_{\mathrm{C}}$. Assuming that over each interstitial sublattice the carbon atoms are randomly distributed, we incorporate the regular configurational entropy. The Gibbs energy function is then written as

$$
\begin{aligned}
G\left(\left\{u_{i}\right\}\right)= & -h_{c} \sum_{i}\left(u_{i}\right)^{2}-2 h_{a} \sum_{i<j} u_{i} u_{j} \\
& +k_{B} T \sum_{i}\left[u_{i} \ln u_{i}+\left(1-u_{i}\right) \ln \left(1-u_{i}\right)\right] .
\end{aligned}
$$

Restricting our study to tetragonal martensite of type 3, we set $u_{1}=u_{2}$ in the following. To study the order-disorder transition between ferrite and tetragonal martensite, two order parameters $X=u_{\mathrm{C}} / 3$ (conservative) and $\eta=\left(u_{3}-u_{1 \text { or } 2}\right) / u_{\mathrm{C}}$ (nonconservative) are classically introduced. $X$ is the average occupation factor of the octahedral sites, while $\eta$ stands for the long-range ordering between sublattice 3 and sublattices 1 and 2 . For ferrite, $u_{1}=u_{2}=u_{3}$ and hence $\eta=0$. For fully ordered martensite, $u_{1}=u_{2}=0$ and $u_{3}=u_{\mathrm{C}}$, and hence $\eta=1$. The intermediate cases $(0<\eta<1)$ represent martensitic crystal structures of prolate tetragonal lattice $(c / a>1)$. Negative values of $\eta,-0.5<\eta<0$, represent oblate tetragonal lattices $(c / a<1)$. The latter case of oblate tetragonality is often overlooked by authors but will prove to be meaningful in the next section. Using the above-defined order parameters, the enthalpy of ordering is written as

$$
H(X, \eta)-H(X, 0)=-9 h_{\eta} X^{2} \eta^{2}
$$

with the alternate strain interaction parameters

$$
\begin{aligned}
h_{X} & =\frac{1}{3}\left(h_{c}+2 h_{a}\right)=\frac{1}{6} \operatorname{tr}(P) \operatorname{tr}(\delta)+\frac{1}{6}\left(P_{a}-P_{c}\right)\left(\delta_{a}-\delta_{c}\right) \\
h_{\eta} & =\frac{2}{3}\left(h_{c}-h_{a}\right)=\frac{2}{3}\left(P_{a}-P_{c}\right)\left(\delta_{a}-\delta_{c}\right) .
\end{aligned}
$$

The enthalpy of ordering is of the form $-3 \lambda_{0} X^{2} \eta^{2}$, where $\lambda_{0}=3 h_{\eta}$ is the ordering energy coefficient. Various values of $\lambda_{0}$ can be found in the literature (Table III). For a given composition $X$, the ordering transition will occur at a critical temperature $T_{c}$. At that temperature, the corresponding order parameter of the ordered phase is noted $\eta_{c}$. As long as $\eta_{c}$ is not zero, the order-disorder transition is of first order.

The Gibbs energy of ordering is a function of $X$ and $\eta$ :

$$
\begin{aligned}
& G(X, \eta)-G(X, 0) \\
& =-9 h_{\eta} X^{2} \eta^{2} \\
& +k_{B} T\left\{\begin{array}{c}
2 X(1-\eta) \ln [X(1-\eta)] \\
+2[1-X(1-\eta)] \ln [1-X(1-\eta)] \\
+X(1+2 \eta) \ln [X(1+2 \eta)] \\
+[1-X(1+2 \eta)] \ln [1-X(1+2 \eta)]
\end{array}\right.
\end{aligned}
$$

In the limit of dilute alloy $(X \ll 1)$, the entropy of the vacancies on the octahedral sites can be neglected. Under this approximation only, the Gibbs energy of ordering exhibits a parabolic dependency on composition $X$, for any given order parameter $\eta$ :

$$
\begin{aligned}
G(X, \eta)-G(X, 0) \approx & -9 h_{\eta} X^{2} \eta^{2} \\
& +k_{B} T X\left[\begin{array}{c}
2(1-\eta) \ln (1-\eta) \\
+(1+2 \eta) \ln (1+2 \eta)
\end{array}\right] .
\end{aligned}
$$

The derivatives of $G$ relative to $\eta$ will be of use in the following sections:

$$
\frac{\partial G}{\partial \eta}=-18 h_{\eta} X^{2} \eta+2 k_{B} T X \ln \left[\frac{1+2 \eta}{1-\eta} \frac{1-X(1-\eta)}{1-X(1+2 \eta)}\right] .
$$

and

$$
\begin{aligned}
\frac{\partial^{2} G}{\partial \eta^{2}}= & -18 h_{\eta} X^{2}+2 k_{B} T X\left[\frac{1}{1-\eta}+\frac{2}{1+2 \eta}\right. \\
& \left.+\frac{X}{1-X(1-\eta)}+\frac{2 X}{1-X(1+2 \eta)}\right] .
\end{aligned}
$$

Since we are interested in supersaturated ferrite up to concentrations of $u_{\mathrm{C}}=0.125$ and more, we will keep the full analytic expressions of the thermodynamic functions, as expressed by the above equations rather than the dilute approximation. This will allow studying the influence of composition on the order-disorder transition. For rough approximations,

TABLE III. Ordering energy coefficient $\lambda_{0}$, in $\mathrm{eV}$, from literature data.

\begin{tabular}{lcccc}
\hline \hline Khachaturyan and Shatalov [3] & Udyansky et al. [6] & Ruban [22] & Clouet et al. [19] & Chentouf et al. [17] \\
\hline 2.73 & 10.77 & 9.5 & 3.00 & 6.24 \\
\hline \hline
\end{tabular}


however, the limiting case of dilute solid solutions is reported below. A first-order development in the vicinity of $X=0$ yields the simplified expression

$$
\frac{\partial G}{\partial \eta} \approx-18 h_{\eta} X^{2} \eta+2 k_{B} T X \ln \left[\frac{1+2 \eta}{1-\eta}\right] .
$$

Compared to the nondilute case, the above expression has lost the composition-ordering coupling terms included in the logarithmic part of Eq. (12). In the following section, the importance of these coupling terms on the critical phenomena will be highlighted.

The functional form of the Gibbs energy of ordering [Eq. (11)] allows for a classical analysis of the order-disorder transition according to Soffa and Laughlin's [23] approach. For given $X$ and $T$, the possible equilibrium order parameters correspond to the extrema of the Gibbs energy of ordering $G(X, \eta)-G(X, 0)$. The relation $\partial G / \partial \eta=0$ thus defines the equilibrium relation $T(X, \eta)$. From Eq. (12), one can check that $\eta=0$ is always an equilibrium value, while the other equilibrium values lie along the $\eta-T$ curve of the implicit equation,

$$
T(X, \eta)=\frac{3 h_{\eta}}{k_{B}} \frac{3 X \eta}{\ln \left[\frac{1+2 \eta}{1-\eta} \frac{1-X(1-\eta)}{1-X(1+2 \eta)}\right]} .
$$

The critical temperature for ordering $T_{c}$ is the temperature above which the disordered state $(\eta=0)$ is more stable than the ordered state $\left(-\frac{1}{2}<\eta<1\right)$. This occurs when $G(X, \eta)=$ $G(X, 0)$. In the dilute approximation, $T(X, \eta)$ is a linear function of $X$, and equilibrium is described by the master curve of the equation

$$
\frac{T(X, \eta)}{T_{c}} \approx \frac{4 \ln 2}{3} \frac{3 \eta}{\ln \left[\frac{1+2 \eta}{1-\eta}\right]},
$$

where $T_{c}$ is a linear function of concentration:

$$
T_{c} \approx \frac{3}{4 \ln 2} \frac{3 h_{\eta}}{k_{B}} X .
$$

The constant $X_{0}=\frac{4 \ln 2}{3}$ in the above formula is a factor close to 1 , which is sometimes omitted in the literature (e.g., [24]).

The ordering curve is represented in Fig. 1 as a solid line. At low temperatures, ordering tends to its maximum with $\eta=1$. When temperature increases, the degree of order decreases down to the critical value $\eta_{c}$ at $T=T_{c}$. In the dilute approximation, $\eta_{c}=0.5$, this state of ordering corresponds to sublattice 3 being two-thirds full of carbon compared to the fully ordered state. Above $T_{c}$, the stable state is full disorder $(\eta=0)$. The equilibrium equation (14) reveals additional features, such as the metastable branch (dashed line in Fig. 1) that goes down to $\eta=-0.5$ at low temperature. This branch accounts for the possibility of metastable stress-free oblate martensite. The maximum temperature on this metastable branch occurs at coordinates $T_{+}=1.010 T_{c}$ and $\eta_{+}=0.377$. The metastable branch intersects the $\eta=0$ axis at temperature $T_{-}=0.924 T_{c}$. At that temperature, the corresponding order parameter on the stable branch is $\eta_{-}=0.716$. The temperatures $T_{-}$and $T_{+}$are related to singularities in the Gibbs energy of ordering (see Fig. 2, top). Actually, at $T=T_{-}$ the derivatives relative to $\eta$ verify $G^{\prime}(0)=G^{\prime \prime}(0)=0$, and at $T=T_{+}, G^{\prime}\left(\eta_{+}\right)=G^{\prime \prime}\left(\eta_{+}\right)=0$. Thus, temperatures $T_{-}, T_{c}$,

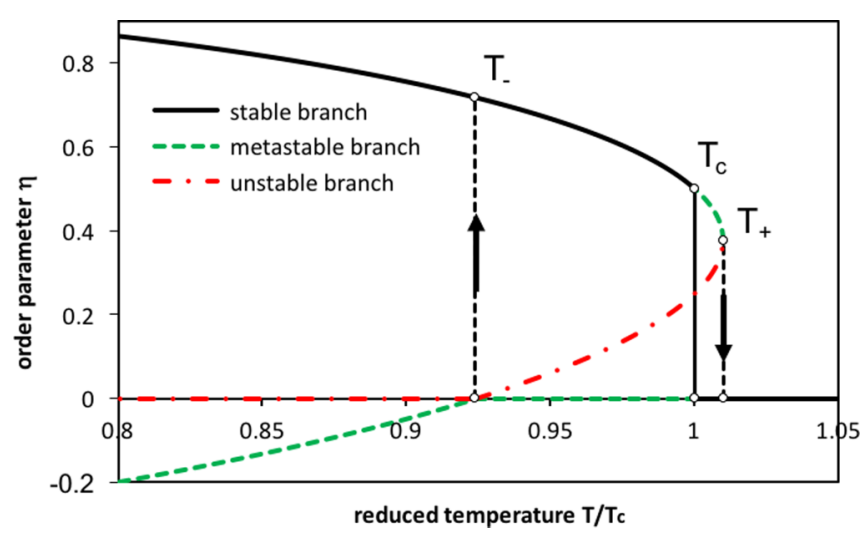

FIG. 1. Ordering curve: order parameter as a function of the reduced temperature. For each temperature $T<T_{+}$, stable, metastable, and unstable equilibria exist. Upon fast heating (cooling) the system will follow the downward (upward) arrow.

and $T_{+}$define intervals of metastability for the ordered and disordered phases:

(1) $T<T_{-}$: order is stable, disorder is unstable;

(2) $T_{-}<T<T_{c}$ : order is stable, disorder is metastable;

(3) $T_{c}<T<T_{+}$: order is metastable, disorder is stable;

(4) $T_{+}<T$ : order is unstable, disorder is stable.

As a consequence, in the temperature range of $T_{-}$to $T_{+}$, two equilibria are possible: one stable and the other metastable. This fact is the origin of the temperature hysteresis effect. Indeed, upon sufficiently fast heating, the system will stick to its metastable ordered state, up to the temperature of $T_{+}$ where the energetic barrier vanishes, and the crystal disorders. A similar behavior will be observed upon fast cooling for the metastable disordered phase, down to temperature $T_{-}$where the crystal will order without energetic barrier.

In the general case of nondilute alloys, the set of equations (10), (12), and (13) must be solved numerically; the critical temperatures, and the corresponding order parameters, become complex functions of composition. However, the abovementioned hysteresis behavior persists.

The transition temperatures $T_{c}, T_{-}$, and $T_{+}$are drawn in Fig. 3 as functions of carbon concentration, using the parameters of Chentouf et al. [19] (Table I) and according to the equations given in the Appendix. At room temperature (300 $\mathrm{K})$, the critical carbon concentration is $x_{\mathrm{C}}=1.14$ at. $\%(0.25$ $\mathrm{wt} \%)$, which is in the range of the experimental data (0.93-2.8 at. \%). At carbon concentration of 11.1 at. \% corresponding to the $\mathrm{Fe}_{8} \mathrm{C}$ compound, $T_{c}=3000 \mathrm{~K}$ and the amplitude of the hysteresis is $T_{+}-T_{-}=246 \mathrm{~K}$.

\section{DISCUSSION}

\section{A. Comparison with molecular dynamics}

The theoretical development described in the previous section allows for a reinterpretation of the molecular dynamics (MD) simulations published by Sinclair et al. [14], in term of hysteresis behavior. The authors used molecular dynamics to study the order-disorder transitions in the Fe-C system. They used Becquart's EAM potential [25], which aims at reproducing most properties of low-carbon $\mathrm{Fe}-\mathrm{C}$ alloys, and 

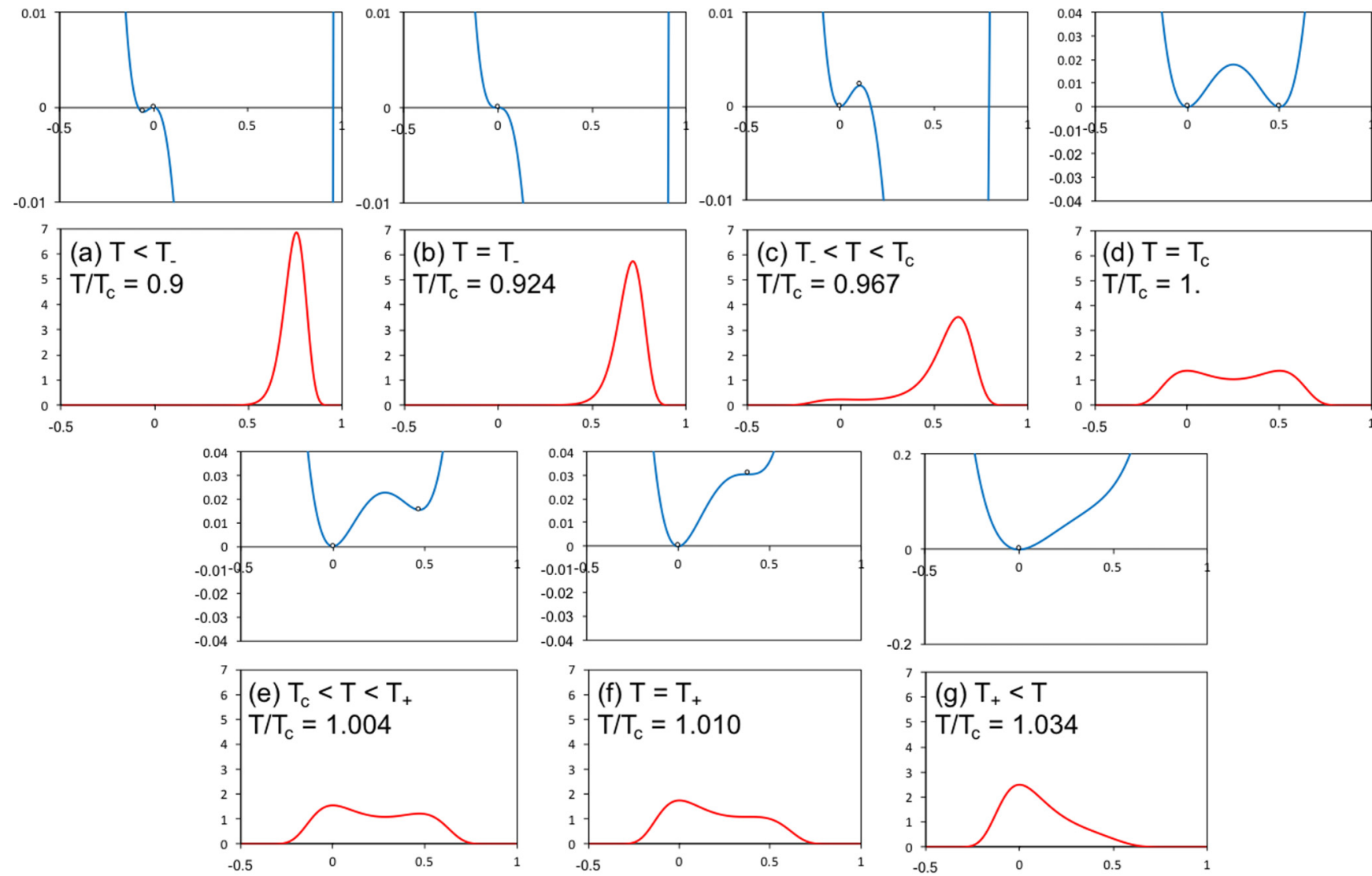

FIG. 2. Top: Gibbs energies of ordering (in $\mathrm{meV} / \mathrm{Fe}$ atom) as a function of the order parameter for $X=0.0147$ at different temperature ranges (enlargements). Bottom: corresponding probability densities for $n_{\mathrm{C}}=250$ carbon atoms (line).

in particular the diffusion mechanism of interstitial carbon. The simulation box of MD simulations contains $n_{\mathrm{C}}=250$ carbon atoms distributed on the octahedral sites of a crystal of $n_{\mathrm{Fe}}=2000$ iron atoms, resulting in the carbon concentration of $u_{\mathrm{C}}=0.125$ (i.e., $x_{\mathrm{C}}=0.111$ ). One of the interesting results of this work is the graph of the carbon occupancies $F_{i}$ as function of temperature (Fig. 4 in [14]). This graph is reported here in Fig. 4, where the original $F_{i}$ have been translated into order parameter $\eta$ via the relation $\eta=F_{3}-\left(F_{1}+F_{2}\right) / 2$.

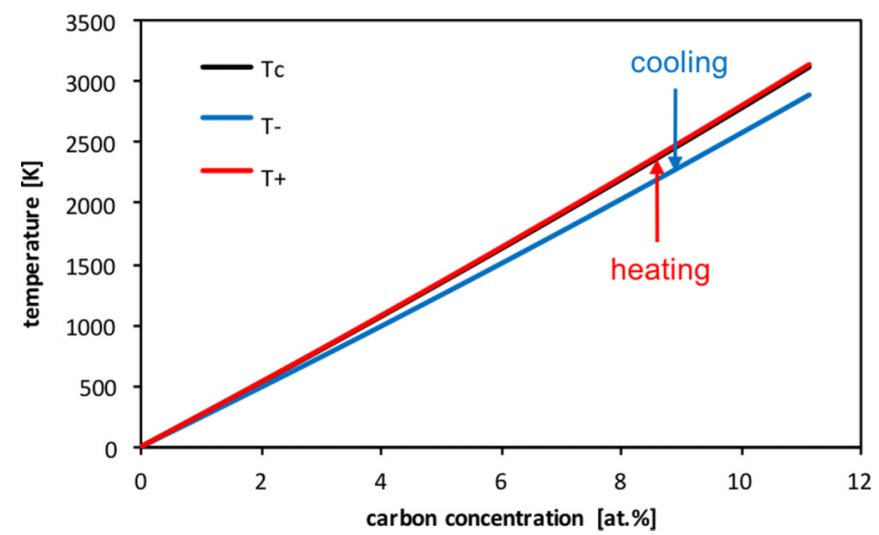

FIG. 3. Critical temperatures $T_{c}$ (black), $T_{-}$(blue), and $T_{+}$(red) as functions of carbon atomic fraction $x_{\mathrm{C}}$. Upon fast heating (cooling) the order-disorder transition starts at temperature $T_{+}\left(T_{-}\right)$.
The ordering curve calculated by the mean-field (MF) model is added in Fig. 4. To allow for quantitative comparison, we have used the parameters corresponding to the potential of the MD simulations, i.e., those determined by Clouet et al. [19] (Table I).

On the graph, the temperature $T$ scans the range of $0.4-1.1$ times the critical temperature. Two sets of points have been retrieved from the MD simulations: The red points correspond to the stage of heating (at $100 \mathrm{~K} / \mathrm{ns}$ ) from the fully ordered low-temperature state, whereas the blue points correspond to cooling $(-80 \mathrm{~K} / \mathrm{ns})$ from the fully disordered high-temperature

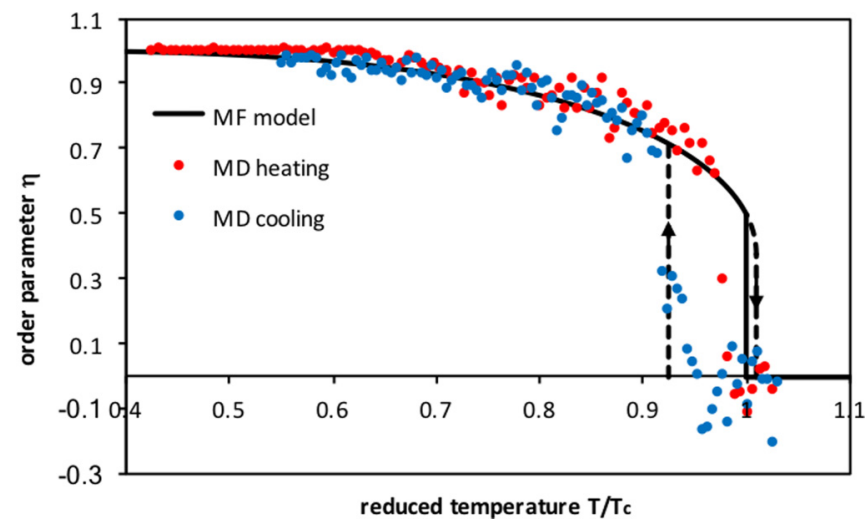

FIG. 4. Ordering curve. Molecular dynamics upon heating (red dots) and cooling (blue dots) compared to mean-field model (lines). 
state. Due to the high heating and cooling rates, the system is expected not to be fully at equilibrium during the whole cycle. Consequently, the curves related to heating and cooling stages do not superimpose exactly, except in two ranges of temperature: $0.65-0.92 T_{c}$ and $0.97-1.03 T_{c}$. For temperatures lower than $0.65 T_{c}$, the points at heating are stuck to $\eta=1$ corresponding to full order. This is probably due to the low diffusivity of carbon in the fully ordered state (see [26]). On the other hand, values in the range $0.92-0.97 T_{c}$ differ significantly: The curve related to heating stays far above the curve related to cooling. Note that the red points follow the stable curve when $T$ increases, while the blue points drop along the $T=T_{-}$vertical line towards the metastable state $\eta=0$. Blue and red points merge around $T=T_{c}$ up to the maximum calculated temperature. On the whole, as long as a stable or metastable equilibrium is reached, the MD points are in very good agreement with the MF curves. Carbon diffusion is thus fast enough for carbon atoms to keep up with the cooling and heating rates, down to the temperature of $0.65 T_{c}$.

The behavior of the system simulated by MD is typical of a hysteresis cycle and can be understood by referring to the ordering curves (Fig. 1) and to the Gibbs energy curves (Fig. 2). During cooling, the system is in the stable state $\eta=0$ down to $T=T_{c}$ [Figs. 2(d)-2(g)]. Under $T_{c}$, the stable state turns to order, but the system remains stuck in the metastable well of the Gibbs energy around $\eta=0$ [Fig. 2(c)]. It gets out of the well towards the ordered state only when temperature $T_{-}$is reached [Figs. 2(a) and 2(b)]. Upon heating, the system stays in the ordered state almost until the temperature $T_{c}$. In large systems, it would probably stay in that state up to the temperature of $T_{+}$when it would get out of the energy well. However, as will be discussed later, thermal fluctuations play a major role in the limited-size system under investigation, and allow the system to get out of its energy well some tens of degrees before the critical point.

From this analysis, we conclude that the MD simulations validate the mean-field model and the hysteresis scheme. This proves that linear elasticity is the predominant source of the thermodynamics of $\mathrm{Fe}-\mathrm{C}$ alloys, at least as far as ordering is concerned. To be complete, it should be mentioned that the critical temperature determined from the MD simulations is about 1.1 times its theoretical mean-field value $(1650 \mathrm{~K}$ vs $1499 \mathrm{~K}$ ). We can thus assess that homogeneous elasticity explains $90 \%$ of the ordering phenomenon. The remaining $10 \%$ probably pertains to thermal expansion effects and shortrange carbon-carbon interaction.

\section{B. Order of the transition}

A quick look at Fig. 4 may suggest that the transition between order and disorder is somehow continuous, and that the transition is thus of second order rather than of first order. This is contradictory with the mean-field model that predicts a first-order transition at any composition, up to $u_{\mathrm{C}}=1.5$ (Fig. 5).

Besides, our analysis reveals that the apparent continuity of the order parameter in MD simulation is the sole effect of thermal fluctuations affecting the limited-size system used to investigate the transition. Indeed, fluctuations of order parameter can be evaluated in the framework of our mean-field

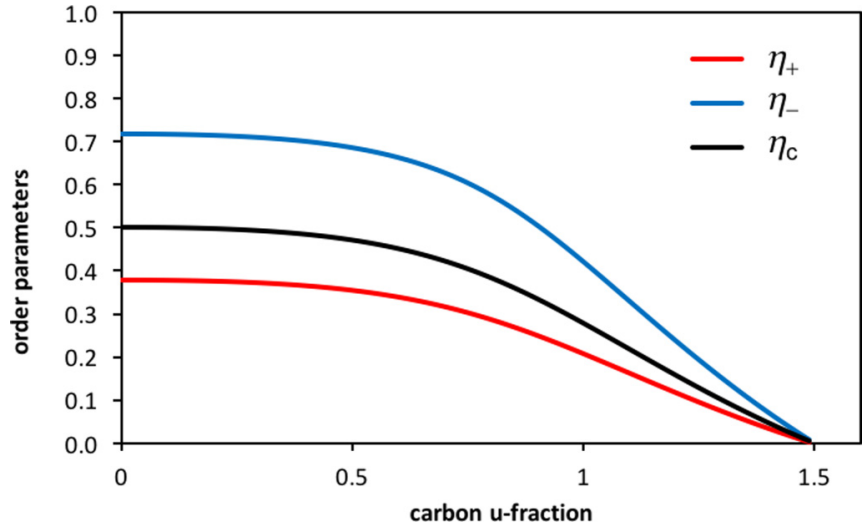

FIG. 5. Order parameters $\eta_{c}$ (black), $\eta_{-}$(blue), and $\eta_{+}$(red) as a function of carbon concentration. Beyond $u_{\mathrm{C}}=1.5, \eta_{c}$ drops to zero and the order-disorder transition becomes of second order.

model. In this purpose, let us recall first that large fluctuations of parameter $\eta$ may arise in situations where the energy barrier between two equilibrium states is low. This happens in two well-documented situations: (i) in the vicinity of a critical point, and (ii) when the Gibbs energy undergoes a change of curvature: $G^{\prime \prime}(\eta)=0$ leads to the squared fluctuation $(\Delta \eta)^{2} \approx 2 k_{B} T / G^{\prime \prime}(\eta)$, to tend to infinity. In our system, case (i) pertains to the temperature region of $T \simeq T_{c}$, where the energy barrier order $\leftrightarrow$ disorder is close to $0.02 \mathrm{meV} / \mathrm{Fe}$-at. [see Fig. 2(d)]. Case (ii) corresponds to $T \gtrsim T_{-}$around $\eta=0$, where the energy barrier disorder $\rightarrow$ order tends to zero [Fig. 2(b)], and to $T \gtrsim T_{+}$around $\eta_{+}=0.377$ where the energy barrier order $\rightarrow$ disorder tends to zero [Fig. 2(f)]. These simple considerations suggest that fluctuations of $\eta$ will be maximum around temperatures $T_{-}, T_{c}$, and $T_{+}$, i.e., in the whole transition region.

A more refined calculation of the fluctuations can be conducted using statistical physics. The probability density of a configuration of order parameter $\eta$ is

$$
\frac{d p(\eta)}{d \eta}=\frac{1}{Z} \exp \left[-\frac{\mathcal{G}(\eta)}{k_{B} T}\right],
$$

where $Z$ is the partition function,

$$
Z=\int_{-1 / 2}^{1} \exp \left[-\frac{\mathcal{G}(\eta)}{k_{B} T}\right] d \eta .
$$

The amplitude of the fluctuations is determined by the spreading of the function $d p / d \eta$. The fluctuations are larger for small systems because the total Gibbs energy of the system is proportional to the number of iron atoms: $\mathcal{G}(\eta)=n_{\mathrm{Fe}} G(\eta)$. Applying Eqs. (18) and (19) to our system, the densities of probability were calculated from the Gibbs energy function of Eq. (10). They are presented in Fig. 2, bottom. It is apparent that the equilibrium fluctuations are limited at low temperature, and large at $T>T_{-}$. In the temperature range $\left[T_{-}, T_{+}\right]$, the metastable equilibrium states have nonvanishing probability of existence. Note that the oblate structures $(\eta<0)$, also visible in Fig. 4, exist at equilibrium as a result of the thermal fluctuations. The fluctuations calculated by the MF model along the stable path (Fig. 6) are very similar to the ones in MD simulations (Fig. 4), which justifies our approach. 


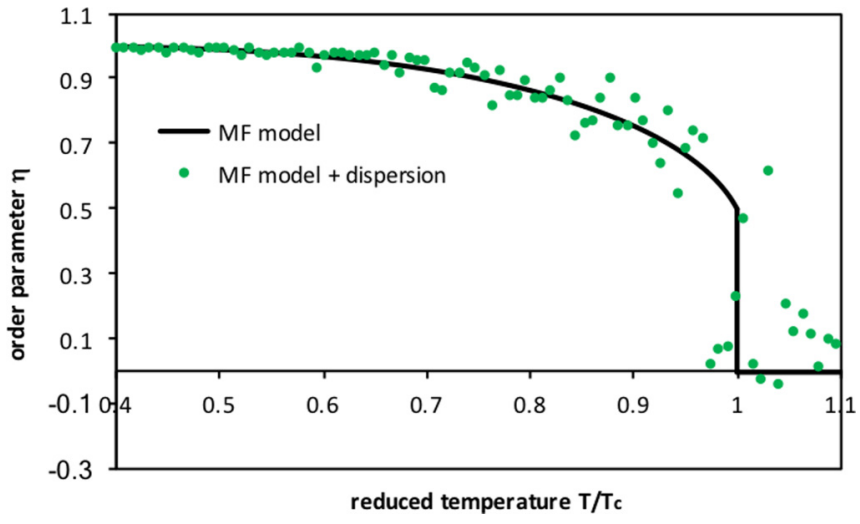

FIG. 6. Stable ordering curve. Mean-field calculations: without dispersion (lines) and with dispersion corresponding to $n_{\mathrm{C}}=250$ carbon atoms (green points).

Our conclusion is that the transition observed by molecular dynamics is indeed of first order, even if apparently blurred by the thermal fluctuations.

An apparent paradox remains in the mean-field model: A first-order transition is expected to be accompanied by a discontinuity of the system volume, which is indeed visible in the work of Sinclair et al. (Fig. 6 in [14]). However, the elasticity theory predicts zero volume change during the transition. In fact, the interstitial carbon atoms induce a dilatation of the crystal:

$$
V=2 V_{\mathrm{Fe}}[1+3 X \operatorname{tr}(\delta)] .
$$

From this equation, the cell volume $V$ appears to be independent of the order parameter $\eta$. This simply results from the fact that the insertion volume $V_{\mathrm{C}}$ of one carbon atom does not depend on the type of site $(1,2$, or 3$)$ it occupies:

$$
V_{\mathrm{C}}=\left(2 \delta_{a}+\delta_{c}\right) V_{\mathrm{Fe}}=8.95 \AA^{3} .
$$

The paradox of zero volume change for a first-order transition is only apparent since, in a deformable system, it is not the volume change that is prescribed to have a finite value by formal thermodynamics, but the change of at least one of the strain components $\varepsilon_{i j}$ of the system [27]. Three strain components are indeed discontinuous across the order-disorder transition, accompanying the cubic to tetragonal change of symmetry of the unit cell. It just happens here that the linear combination $\sum \varepsilon_{i i}$ is null, leading to $\Delta V=0$, at least to the first order. We conclude that the volume change observed in MD simulations probably arises from the short-range carbon-carbon interactions.

\section{SUMMARY}

A mean-field model based on the elasticity theory of point defects was developed. From the Gibbs energy function of disordered $\alpha$-Fe and partially ordered $\alpha^{\prime}$-Fe, the temperature hysteresis of the order-disorder transition was evidenced. Its origin is shown to be thermodynamic. The major consequence of this hysteresis is the downward shift in temperature of the disorder to order transition during cooling of supersaturated $\alpha$-Fe. For the composition of $x_{\mathrm{C}}=11.1$ at. \% corresponding to compound $\mathrm{Fe}_{8} \mathrm{C}$, the shift rises to about $250 \mathrm{~K}$. These findings are quantitatively confirmed by molecular dynamics simulations. Furthermore, we assess that the order-disorder transition is of first order, although the transition viewed by molecular dynamics is apparently continuous, and the volume variation associated to the transition is predicted to be null. Unambiguously, this work also shows that elasticity is the predominant source of the thermodynamics of the Fe-C solid solutions.

\section{ACKNOWLEDGMENT}

The Agence Nationale de la Recherche (Contract No. SPIDERMAN 13-BS08-0014-02) is thanked for its financial support. Enlightening discussions with C. Sinclair and M. Perez are gratefully acknowledged.

\section{APPENDIX: BEYOND THE DILUTE APPROXIMATION}

Beyond the approximation of the dilute solid solution, temperatures $T_{c}, T_{-}$, and $T_{+}$are no longer linear functions of composition. It results that the shape of the ordering curve is only approximately given by the master curve of Fig. 1. A secondorder approximation of $T_{c}$ and $T_{+}$, with a maximum relative error of $0.1 \%$ in the range of $X$ from 0 up to 0.0147 is written as

$$
T_{c} \approx \frac{3 h_{\eta}}{k_{B}} \frac{X}{0.924}\left(1-\frac{X}{0.924}\right),
$$

and

$$
T_{+} \approx \frac{3 h_{\eta}}{k_{B}} \frac{X}{0.915}\left(1-\frac{X}{0.915}\right)
$$

with the constant $\frac{4}{3} \ln 2 \approx 0.924$. Besides, the exact value of $T_{-}$is

$$
T_{-}=\frac{3 h_{\eta}}{k_{B}} X(1-X)
$$

This last function is not to be mistaken with the critical temperature $T_{c}$.
[1] W. L. Fink and E. D. Campbell, Trans. Am. Soc. Steel Treat. 9, 717 (1926).

[2] C. Zener, Phys. Rev. 74, 639 (1948).

[3] A. G. Khachaturyan and G. V. Shatalov, Fiz. Met. Metalloved. 32, 5 (1971).

[4] G. V. Kurdjumov and A. G. Khachaturyan, Acta Metall. 23, 1077 (1975).
[5] A. Taylor and M. Cohen, Prog. Mater. Sci. 36, 225 (1992).

[6] A. Udyansky, J. von Pezold, A. Dick, and J. Neugebauer, Phys. Rev. B 83, 184112 (2011).

[7] L. Xiao, Z. Fan, Z. Jinxiu, Z. Mingxing, K. Mokuang, and G. Zhenqi, Phys. Rev. B 52, 9970 (1995).

[8] D. A. Mirzaev, A. A. Mirzoev, and P. V. Chirkov, Metall. Mater. Trans. A 47, 637 (2016). 
[9] J. Epp, T. Hirsh, and C. Curfs, Metall. Mater. Trans. A 43, 2210 (2012).

[10] S. Y. P. Allain, G. Geandier, J. C. Hell, M. Soler, F. Danoix, and M. Gouné, Scr. Mater. 131, 15 (2017).

[11] S. Nagakura, Y. Hirotsu, M. Kusunoki, T. Suzuki, and Y. Nakamura, Metall. Trans. A 14, 1025 (1983).

[12] Z. Fan, L. Xiao, Z. Jinxiu, K. Mokuang, and G. Zhenqi, Phys. Rev. B 52, 9979 (1995).

[13] O. Sherby, J. Wadsworth, D. Lesuer, and S. Syn, Mater. Sci. Forum 539-543, 215 (2006).

[14] C. W. Sinclair, M. Perez, R. G. A. Veiga, and A. Weck, Phys. Rev. B 81, 224204 (2010).

[15] M. Hillert, J. Alloys Compd. 320, 161 (2001).

[16] D. Kandaskalov and P. Maugis, Comput. Mater. Sci. 128, 278 (2017).

[17] S. Chentouf, S. Cazottes, F. Danoix, M. Gouné, H. Zapolsky, and P. Maugis, Intermetallics 89, 92 (2017).
[18] D. J. Bacon, Scr. Metall. 3, 735 (1969).

[19] E. Clouet, S. Garruchet, H. Nguyen, M. Perez, and C. S. Becquart, Acta Mater. 56, 3450 (2008).

[20] L. Cheng, A. Bottger, Th. H. de Keijser, and E. J. Mittemeijer, Scr. Metall. 24, 509 (1990).

[21] S. B. Ren and S. T. Wang, Metall. Trans. A, 19, 2427 (1988).

[22] A. Ruban, Phys. Rev. B 90, 144106 (2014).

[23] W. A. Soffa and D. E. Laughlin, Acta. Metall. 37, 3019 (1989).

[24] A. Udyansky, J. von Pezold, V. N. Bugaev, M. Friák, and J. Neugebauer, Phys. Rev. B 79, 224112 (2009).

[25] C. S. Becquart, J. M. Raulot, G. Bencteux, C. Domain, M. Perez, S. Garruchet, and H. Nguyen, Comput. Mater. Sci. 40, 119 (2007).

[26] B. Lawrence, C. W. Sinclair, and M. Perez, Modell. Simul. Mater. Sci. Eng. 22, 065003 (2014).

[27] H. B. Callen, Thermodynamics and an Introduction to Thermostatics, 2nd ed. (John Wiley, New York, 1960), p. 237. 